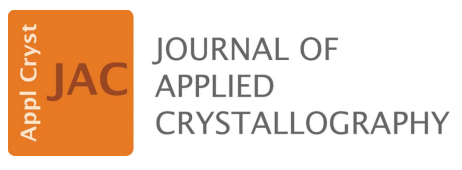

ISSN 1600-5767

Received 27 August 2019

Accepted 23 December 2019

Edited by S. Boutet, SLAC National Accelerator Laboratory, Menlo Park, USA

Keywords: Bragg coherent X-ray diffraction imaging; Au nanoparticles; energy scanning.

Supporting information: this article has supporting information at journals.iucr.org/j

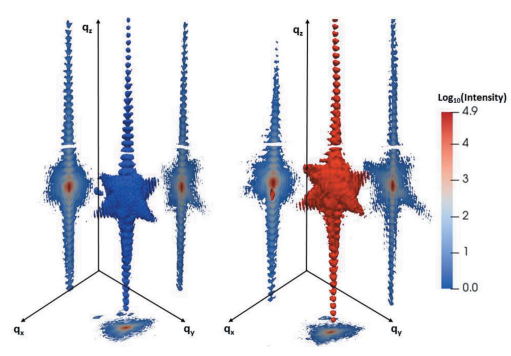

C 2020 International Union of Crystallography

\section{Multi-wavelength Bragg coherent X-ray diffraction imaging of Au particles}

\author{
F. Lauraux, ${ }^{a}$ T. W. Cornelius, ${ }^{a *}$ S. Labat, ${ }^{a}$ M.-I. Richard, ${ }^{a, b}$ S. J. Leake, ${ }^{b}$ T. Zhou, \\ O. Kovalenko, ${ }^{c}$ E. Rabkin, ${ }^{c}$ T. U. Schülli ${ }^{b}$ and O. Thomas ${ }^{a}$
}

\footnotetext{
${ }^{\mathbf{a}}$ Aix Marseille Université, Université de Toulon, CNRS, IM2NP, Marseille, France, ${ }^{\mathbf{b}}$ ID01/ESRF - The European Synchrotron, 71 Avenue des Martyrs, 38000 Grenoble, France, and ${ }^{\mathrm{c}}$ Department of Materials Science and Engineering, Technion - Israel Institute of Technology, 32000 Haifa, Israel. *Correspondence e-mail: thomas.cornelius@im2np.fr
}

Multi-wavelength (mw) Bragg coherent X-ray diffraction imaging (BCDI) is demonstrated on a single Au particle. The multi-wavelength Bragg diffraction patterns are inverted using conventional phase-retrieval algorithms where the dilation of the effective pixel size of a pixelated 2D detector caused by the variation of the X-ray beam energy is mitigated by interpolating the raw data. The reconstructed Bragg electron density and phase field are in excellent agreement with the results obtained from conventional rocking scans of the same particle. Voxel sizes of about $6^{3} \mathrm{~nm}^{3}$ are obtained for reconstructions from both approaches. Phase shifts as small as $0.41 \mathrm{rad}$, which correspond to displacements of $14 \mathrm{pm}$ and translate into strain resolution better than $10^{-4}$ in the $\mathrm{Au}$ particle, are resolved. The displacement field changes shape during the experiment, which is well reproduced by finite element method simulations considering an inhomogeneous strained carbon layer deposited on the $\mathrm{Au}$ particle over the course of the measurements. These experiments thus demonstrate the very high sensitivity of BCDI and mw-BCDI to strain induced by contaminations. Furthermore, mw-BCDI offers new opportunities for in situ and operando 3D strain imaging in complex sample environments.

\section{Introduction}

In the past two decades, coherent X-ray diffraction imaging (CDI) has made tremendous progress facilitating the 3D mapping of nanostructured samples (Miao et al., 1999; Thibault et al., 2006; Nishino et al., 2009). This method retrieves the sample scattering function from a coherent X-ray diffraction data set using computational inversion algorithms to determine the phase of the scattered wave, which is not directly measurable by a detector (Fienup, 1978, 1982; Marchesini et al., 2003). In the Bragg condition, the retrieved phase is directly related to the displacement field and thus the strain within a crystal. The first Bragg CDI (BCDI) experiments concentrated on almost perfect and strain-free samples, such as $\mathrm{Au}$ or $\mathrm{Pb}$ crystals deposited on a substrate (Williams et al., 2003; Robinson et al., 2001; Pfeifer et al., 2006). With further progress of the experimental techniques as well as of the phase-retrieval algorithms, determination of the complete strain tensor from BCDI of six independent Bragg peaks on a single $\mathrm{ZnO}$ nanorod was demonstrated, rendering a spatial resolution of $40 \mathrm{~nm}$ (Newton et al., 2010). The aforementioned measurements all concentrated on weakly deformed objects without any defects. Following the imaging of a dislocation in Si by coherent X-ray diffraction reported by Jacques et al. (2011), nanostructures containing a single defect or high strain came into focus (Favre-Nicolin et al., 2009, 2010; Labat et al., 2015; Clark et al., 2015; Ulvestad et al., 2015a; Diaz et al., 2009; 
Biermanns et al., 2009; Minkevich et al., 2007). Recently, Labat et al. (2015) demonstrated the reconstruction of GaN nanowires that contain inversion domain boundaries by measuring five independent Bragg peaks of the same nanorod, rendering a precision of $1 \mathrm{pm}$ in the displacement field and a spatial resolution of $10 \mathrm{~nm}$. Recent developments aim to image defects as well as the strain field in nanostructures in situ and operando. The entire network of dislocations within an individual calcite crystal and their propagation during repeated growth and dissolution cycles demonstrate the potential of BCDI for studying the mechanisms underlying the response of crystalline materials to external stimuli, as reported by Clark et al. (2015). Similarly, Dupraz et al. (2017) imaged for the first time a prismatic dislocation loop in an Au crystal induced by nano-indentation, and Ulvestad et al. (2015b) reported the evolution of strain in a single particle of $\mathrm{LiNi}_{0.5} \mathrm{Mn}_{1.5} \mathrm{O}_{4}$, which is used in lithium ion batteries, during charging and discharging.

In $\mathrm{BCDI}$, one records the $3 \mathrm{D}$ intensity distribution around a Bragg peak by a variation of the incident angle of the X-ray beam with respect to the sample on the order of $1^{\circ}$. Given the often nanometric size of the sample, it is rarely mounted in the exact centre of rotation of a diffractometer. Since typical stateof-the-art high-resolution diffractometers exhibit a wobble, i.e. an eccentricity, of about $6 \mu \mathrm{m}$ over a full rotation, there is a risk of nanostructures moving out of the nano-focused hard $\mathrm{X}$-ray beam during rocking scans. The sample thus either has to be realigned with respect to the focused X-ray beam after a certain amount of rotation or has to be simultaneously translated along the beam direction to compensate for prior misalignments and the diffractometer wobble. These compensations and realignments increase the measurement time considerably and may result in artefacts in the retrieved phase caused by the fact that the nanostructure is illuminated by different parts of the focused X-ray beam which may not have a perfectly plane wavefront. The latter issue can be solved by Bragg ptychography. In addition, movements of diffractometer motors during rocking scans and additional lateral alignments of the sample stage risk generating vibrations that are detrimental to sample environments such as nano-mechanical testing devices, where vibrations may lead to damage to the nanostructure under load (Dupraz et al., 2017; Ren et al., 2014). These problems can be circumvented by scanning the energy of the incident X-ray beam as demonstrated by Cornelius et al. (2012) and Davydok et al. (2018) during the mechanical deformation of $\mathrm{SiGe}$ islands and $\mathrm{Au}$ nanowires, respectively. Kirkpatrick-Baez (KB) mirrors are achromatic, the position of the X-ray beam focus on the sample surface is constant as a function of energy, and thus the beam spot on the sample remains stationary for the entire measurement. However, the change in energy complicates the reconstruction of the lost phase. The problem arises from the fact that a change in X-ray energy affects the apparent size in reciprocal space as well as the absorption/detection efficiency of a pixel in a pixelated detector. While the latter can typically be neglected or corrected easily, the interpolation of the apparent pixel size within the used energy range creates major complications. Recently, Cha et al. (2016) demonstrated the reconstruction of an $\mathrm{Au}$ particle measured by multiwavelength (mw) BCDI. The authors reconstructed the 3D direct space image on a slice-by-slice basis, thus taking into account the wavelength-dependent pixel size.

Here, we demonstrate BCDI and reconstruction of both shape and phase of a selected Au particle measured by rocking scans and by scanning the energy of the incident X-ray beam. Instead of reconstructing the 3D image on a slice-by-slice basis, the Bragg electron density and the phase are directly reconstructed from the raw diffraction intensities without any interpolation. The results obtained using this simplified approach are in excellent agreement with reconstructions obtained from rocking scans recorded on the same object.

\section{Experimental}

$\mathrm{Au}$ particles were obtained by solid-state dewetting of a $30 \mathrm{~nm}$ thin Au film on a $c$-plane oriented polished sapphire substrate (Kovalenko et al., 2013). The film was electron-beam evaporated on a lithographically patterned substrate consisting of $\sim 2 \mu \mathrm{m}$-sized holes in a marked $50 \mu \mathrm{m}$ pitch grid. The samples were annealed in ambient air at $1173 \mathrm{~K}$ for $24 \mathrm{~h}$, resulting in the agglomeration of the patterned thin film and the formation of single-crystalline faceted Au particles. The patterned thin film eventually results in a regular array of crystals with a single and isolated particle in the centre of each square as illustrated by the scanning electron micrographs presented in Figs. 1(a) and 1(b). All obtained crystallites exhibit the same well defined out-of-plane orientation with the $\mathrm{Au}$ [111] direction normal to the (0001) sapphire surface as illustrated by the top view of an isogonal truncated octahedron [Fig. 1(c)] (which is the equilibrium shape of the Au particles; Kovalenko \& Rabkin, 2015) and the pole figure [Fig. 1(d)] obtained from the Bragg coherent X-ray diffraction pattern (BCDP) presented in Fig. 4(a) (Richard et al., 2018).

BCDI of Au particles was performed at the ID01 beamline at the European Synchrotron Radiation Facility (ESRF), Grenoble, France (Leake et al., 2019). Here, the incident X-ray photon energy was set to $9 \mathrm{keV}$ using an $\mathrm{Si}(111)$ double-crystal monochromator and the X-ray beam was focused by a pair of KB mirrors. A set of slits located in front of the focusing optics were closed down to an aperture of $50 \mu \mathrm{m}$ (vertically) by $30 \mu \mathrm{m}$ (horizontally). The focal profile of the beam was characterized using a 2D ptychography (Pfeiffer, 2018) approach on a test pattern featuring a $30 \mu \mathrm{m}$-diameter tungsten Siemens star placed close to the focal position of the KB mirrors (Leake et al., 2017). The W structure and the complexvalued wavefront were retrieved simultaneously using the ptychography reconstruction code of the PyNX package (Mandula et al., 2016). A cut of the retrieved experimental complex illumination is displayed in Fig. 2(a), showing a Gaussian beam waist with focal spot dimensions of $550 \mathrm{~nm}$ (vertical) and $530 \mathrm{~nm}$ (horizontal) [see Fig. 2(b)]. The diffracted X-rays were recorded with a 2D MAXIPIX pixel detector with a pixel size of $55 \times 55 \mu \mathrm{m}$ installed $1 \mathrm{~m}$ downstream from the sample position (Ponchut et al., 2011). This 
distance was chosen so that the diffraction pattern was sufficiently oversampled (by a factor of 4, 6 and 6 for $x, y$ and $z$, respectively).

The $\mathrm{Au}$ particles were located by the quick-mapping approach, scanning an extended area of several tens of square micrometres of the sample through the focused X-ray beam and recording the diffraction yield of the $\mathrm{Au} 111$ Bragg reflection (Chahine et al., 2014, 2015). Once an individual well separated $\mathrm{Au}$ particle was located, diffraction patterns were recorded both by rocking scans and by scanning the energy of the incident X-ray beam. Schematics of the two scanning approaches are presented in Fig. 3. During rocking scans the incident angle $\omega$ was varied by $\pm 1^{\circ}$ (covering 1.37, 1.15 and $0.99 \mathrm{~nm}^{-1}$ in $q_{x}, q_{y}$ and $q_{z}$, respectively) in steps of $0.005^{\circ}$. For mw-BCDI, the energy was scanned from 8.75 to $9.25 \mathrm{keV}$ in steps of $2 \mathrm{eV}$. After each $10 \mathrm{eV}$ of change in energy, the undulator gaps were readjusted to keep the incident intensity $I_{0}$ constant over the scanned energy range. The fact that the diffraction signal is probed along $q_{z}$ during energy scans limits the information within the $q_{x} q_{y}$ plane. Therefore, the $2 \theta$ angle of the detector was varied simultaneously by $\pm 1.0^{\circ}$ in steps of 8 millidegrees, thus eventually probing the reciprocal space in the same manner as during rocking scans (schematically shown in Fig. 3), covering 1.00, 1.07 and $1.01 \mathrm{~nm}^{-1}$ in $q_{x}, q_{y}$ and $q_{z}$, respectively. The volumes probed in reciprocal space by the two aforementioned methods are thus comparable, with the multi-wavelength approach examining about $20 \%$ less in $q_{x}$ and in $q_{y}$ than a rocking scan. Such practical considerations depend also on the orientation of the probed Bragg peak. The measurement time for rocking scans and energy scans including the scan of the detector amounts to about 15 and $45 \mathrm{~min}$, respectively, at similar exposure times to radiation (i.e. similar dose). The increased measurement time for mw-BCDI compared with ordinary BCDI originates from the adjustment of the undulator gaps, which is rather time consuming.

The reconstructions of the Bragg electron density and the phase from the BCDPs were obtained using the CDI algorithms in the $P y N X$ package; the same input parameters were used for both types of measurements. The fact that the incident beam is not fully coherent was taken into account by
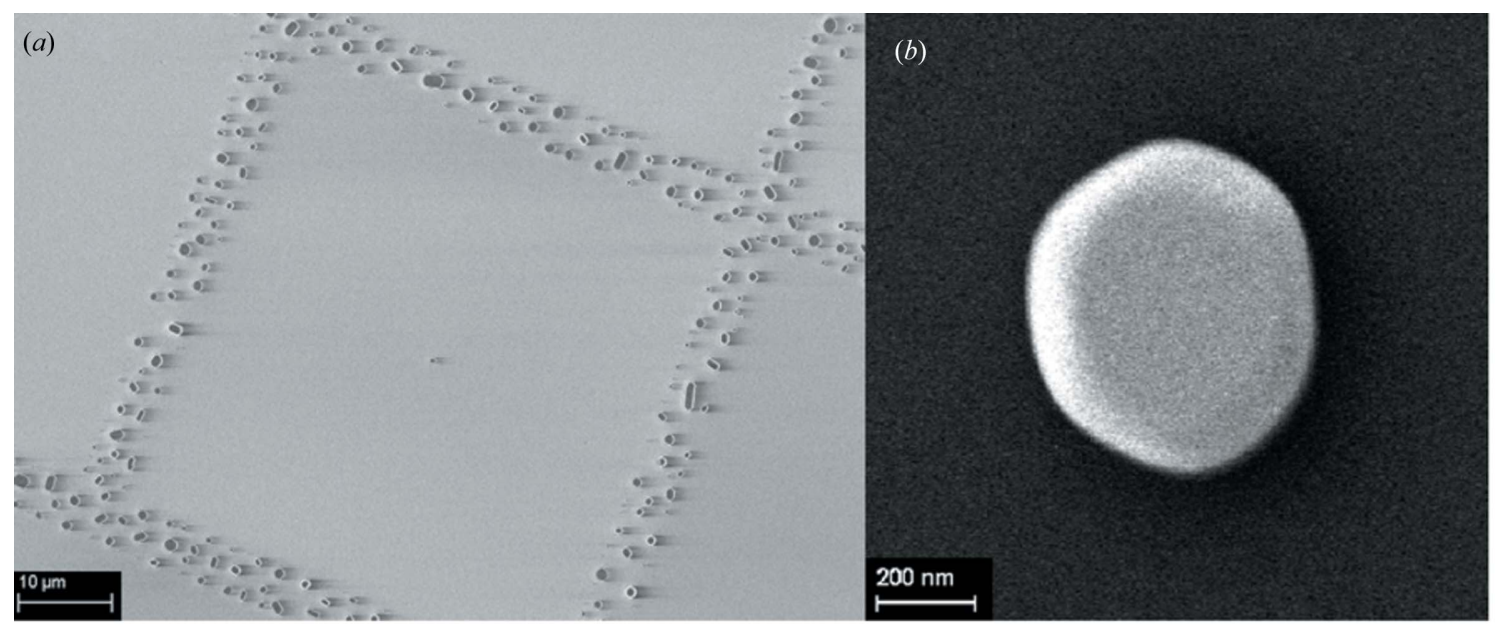

(c)
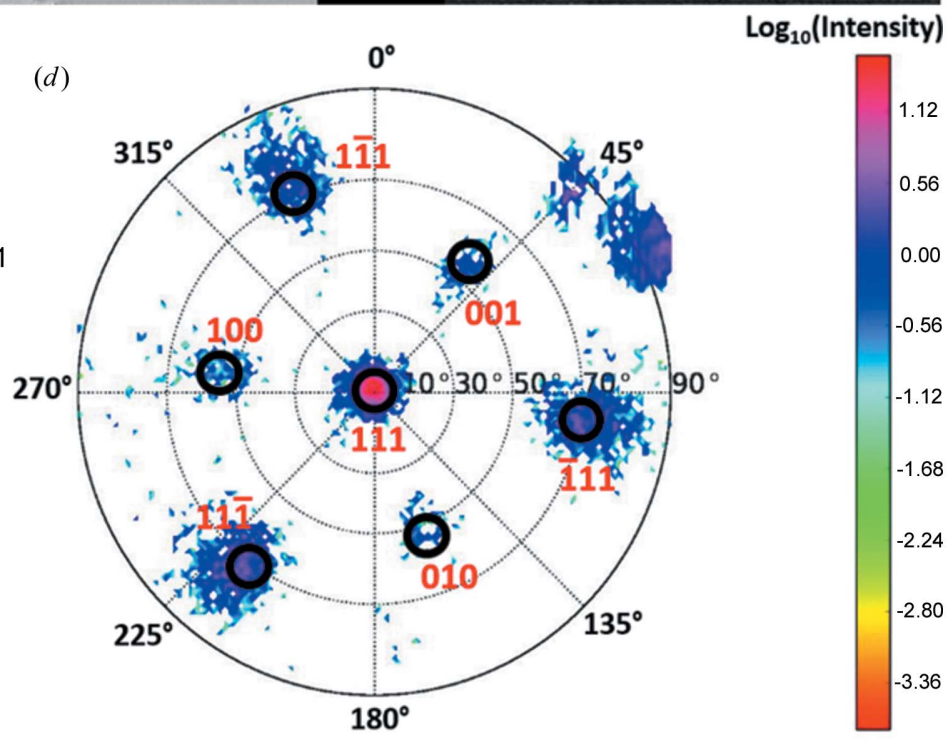

Figure 1

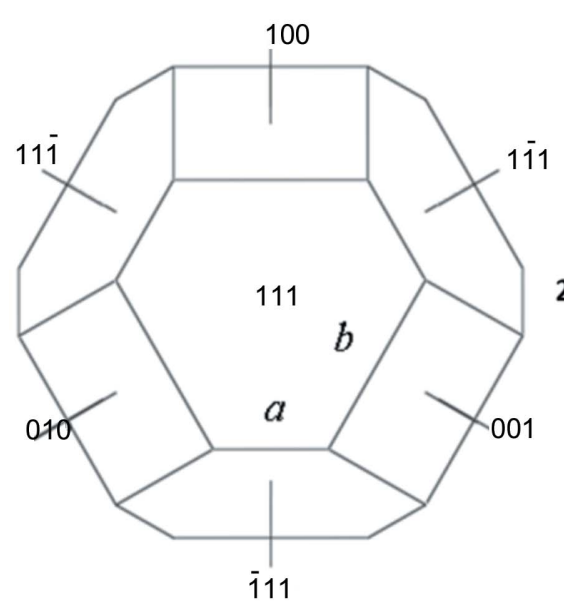

Scanning electron microscopy images of $(a)$ a patterned square with an isolated Au particle in the centre and $(b)$ an isolated Au particle. $(c)$ Top view of an isogonal truncated octahedron (Kovalenko \& Rabkin, 2015). (d) Pole figure of the 3D diffraction pattern presented in Fig. 4, illustrating the facet orientations (Richard et al., 2018). 

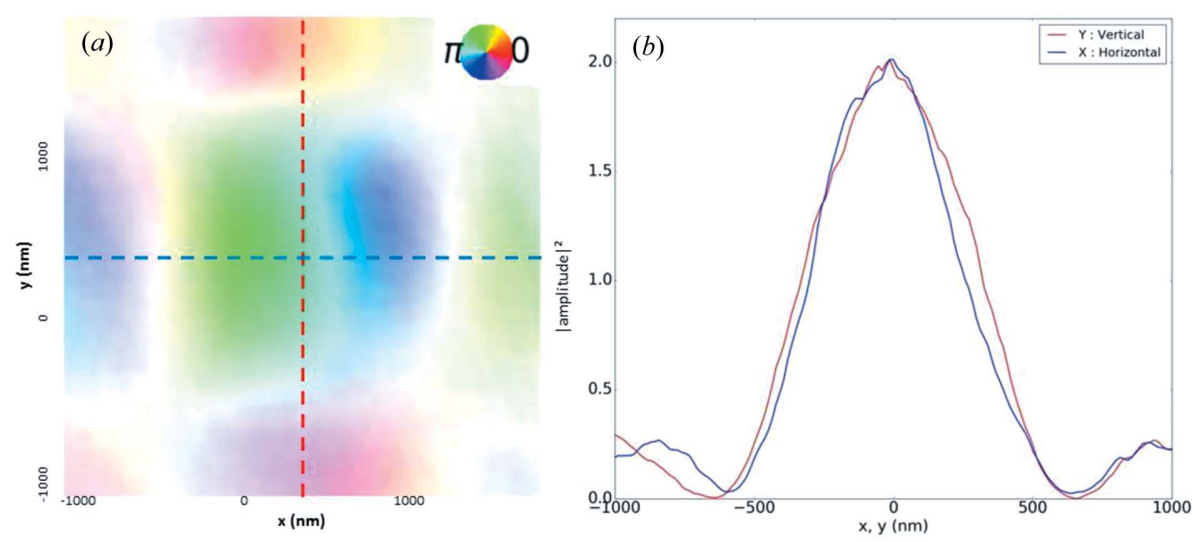

Figure 2

(a) Experimental complex illumination at the focal plane of the Kirkpatrick-Baez mirrors and $(b)$ line profiles across the complex illumination showing Gaussian profiles with sizes of 550 and $530 \mathrm{~nm}$ in the vertical and horizontal directions, respectively.
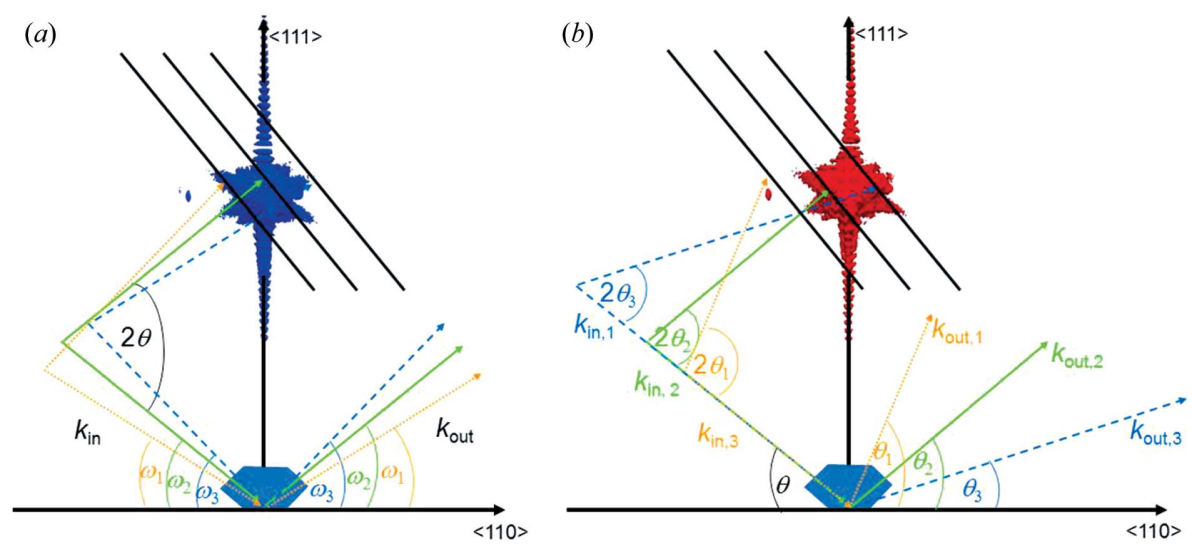

Figure 3

Schematics of the detector movement in reciprocal space for $(a)$ classic BCDI and $(b)$ mw-BCDI combined with an angular displacement of the detector in $2 \theta$. Both approaches probe similar volumes in reciprocal space in exactly the same way.

using a partial coherence point-spread function. Intensity auto-correlation with a relative threshold of 0.1 was applied as initial support. The Hermitian symmetry of the complex scattered amplitude leads to the reconstruction of two conjugated objects that are equivalent. To bias the algorithm towards one solution, the support was cut in half during 10 cycles. The support was updated every 20 cycles with a relative threshold of 0.18 and the absolute threshold was obtained by averaging the values taken over the support volume. It was further updated by convoluting the object amplitude with a Gaussian with a size exponentially decreasing from 2 to 1 . The algorithms used first were 800 RAAR (relaxed averaged alternating reflections; Luke, 2005) followed by 150 ER (error reduction; Fienup, 1978; Gerchberg \& Saxton, 1972). The only difference in the treatment of the two types of measurements is the implementation of a support post-expansion in the case of energy scans. This method, which first expands the support by two pixels then shrinks it by four pixels and finally reexpands it by two, eliminates artefacts appearing outside of the reconstructed object as illustrated in Fig. S1 of the supporting information. These artefacts, which exist only for energy scans and not for rocking scans, might be caused by the dilation of the effective pixel size.

Finite element method (FEM) simulations of the displacement field in an $\mathrm{Au}$ crystal covered by an inhomoge-

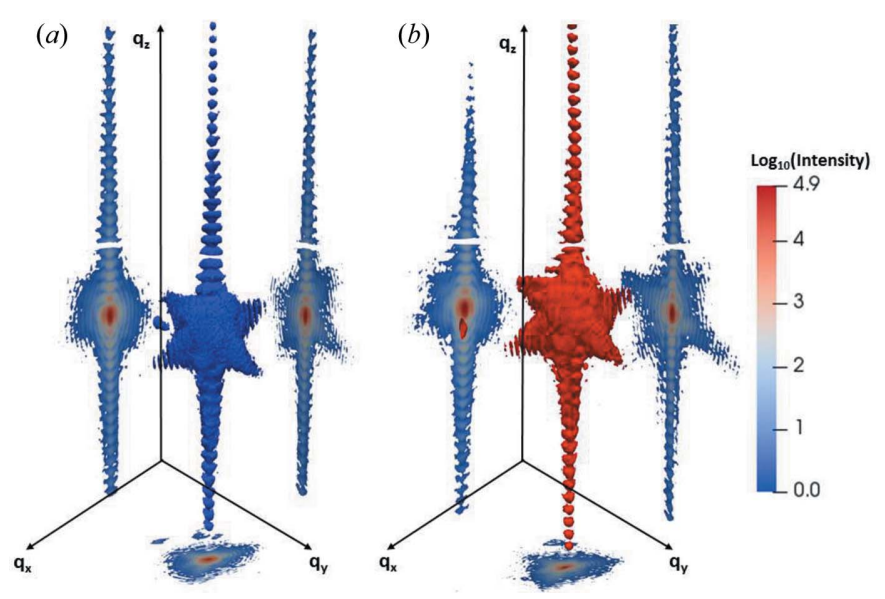

Figure 4

3D iso-surfaces of the diffraction intensity in the vicinity of the Au 111 Bragg peak as well as three orthogonal cuts through reciprocal space recorded by $(a)$ classic BCDI and $(b)$ mw-BCDI for the same Au particle. neous strained carbon layer were performed using COMSOL Multiphysics. The computations took into account the exact shape of the Au crystal determined by BCDI and the complete stiffness tensor $C_{\alpha \beta}$ considering the values for bulk $\mathrm{Au}\left(C_{11}=\right.$ $192 \mathrm{GPa}, C_{12}=163 \mathrm{GPa}$ and $C_{44}=42 \mathrm{GPa}$ ) (Neighbours \& Alers, 1958). The carbon layer and the sapphire substrate were treated as isotropic materials with Young's moduli of 4.3 and $400 \mathrm{GPa}$, respectively. For the calculations, fixed boundary conditions were applied on the lower facet of the substrate.

\section{Results and discussion}

Three-dimensional BCDI diffraction patterns of an $\mathrm{Au}$ particle and three orthogonal cuts through reciprocal space recorded both by a rocking curve and by scanning the incident X-ray energy are presented in Figs. $4(a)$ and 4(b), respectively. The two patterns are very similar, showing clear facet streaks 
and well defined finite-size fringes, which indicate a strain-free or very weakly strained crystal.

The Bragg electron density and the phase were reconstructed for both diffraction patterns using raw data, as well as interpolated data, to study the influence of interpolations and possible artefacts induced by them. The results are presented in Fig. 5. In the case of reconstructions from the raw data, interpolation was only applied after the phase-retrieval process in order to orthonormalize the results obtained. As demonstrated in Figs. 5( $a$ ) and 5(b), post-interpolation induces a pixelated crystal shape due to an increased voxel size of about $13 \mathrm{~nm}$ as well as artefacts in the phase at the edges of the reconstructed crystal. These shortcomings can be circumvented by pre-interpolation, mitigating the dilation of the effective pixel size caused by the variation of the photon energy as illustrated in Figs. $5(c)$ and $5(d)$. Here, the voxel sizes amount to $4.93 \times 6.13 \times 6.38 \mathrm{~nm}$ and $6.65 \times 5.51 \times$ $6.12 \mathrm{~nm}$ for classic BCDI and mw-BCDI, respectively.
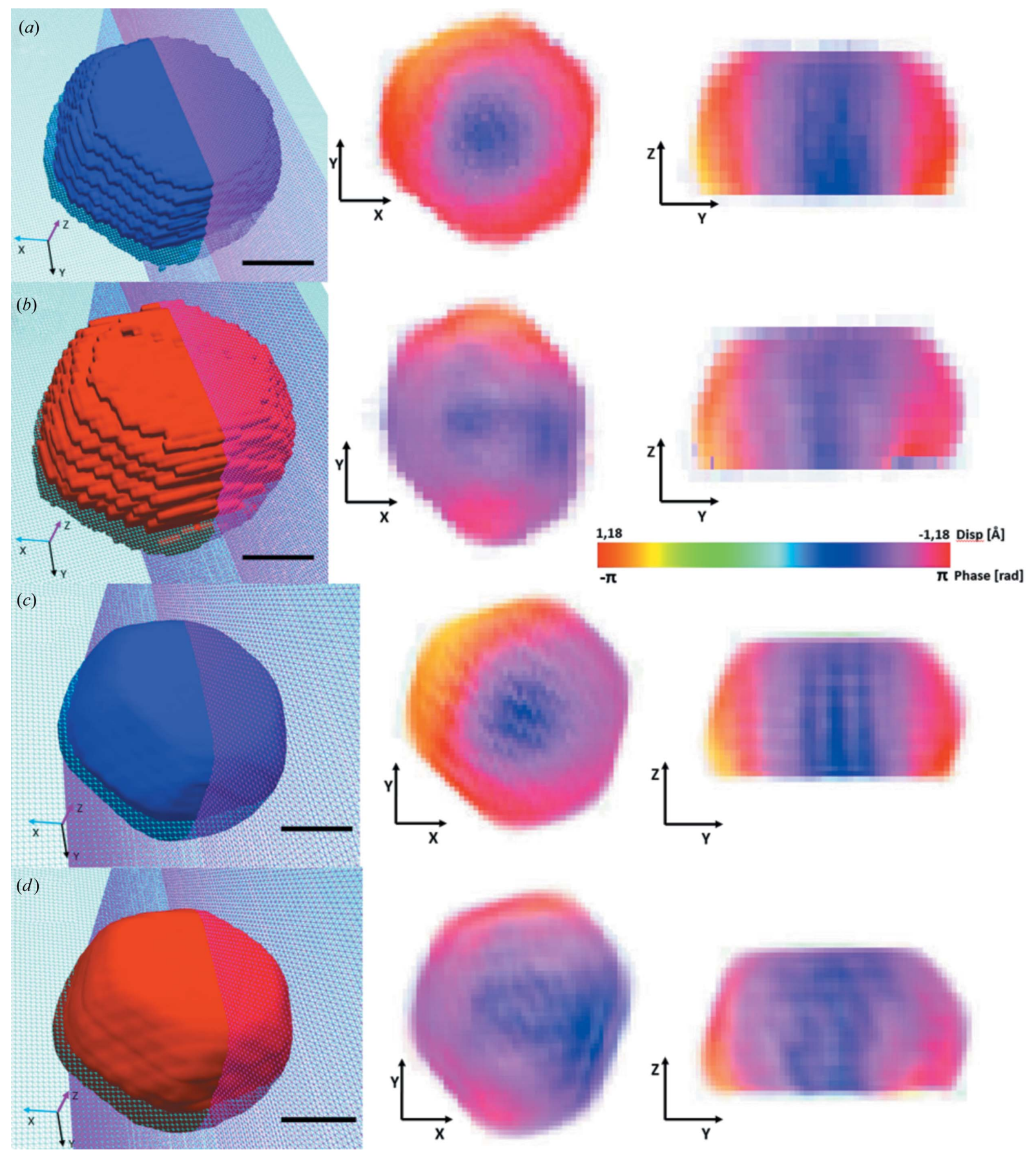

Figure 5

3D reconstruction of the Bragg electron density and $y x$ and $z y$ slices of the reconstructed phase for a post-interpolated $(a) \mathrm{BCDP}$ and $(b)$ mw-BCDP and for a pre-interpolated $(c) \mathrm{BCDP}$ and $(d)$ mw-BCDP. Scale bars are $150 \mathrm{~nm}$ in length. 
However, pre-interpolation induces stripe-like artefacts in the retrieved phase, particularly for data obtained by rocking scans.

Despite the aforementioned imperfections, the reconstructed Bragg electron density and phase are essentially the same for both rocking scans and energy scans, regardless of whether or not interpolation was executed before or after phase retrieval. The reconstructed shape of the Au crystal shows a well faceted particle with large $\{111\}$ and $\{100\}$ facets as illustrated by the pole figure displayed in Fig. 1(d) (Richard et al., 2018), which was obtained from the BCDP shown in Fig. 4(a), and in agreement with the isogonal truncated octahedron shown in Fig. 1(c). It has a lateral size of $550 \mathrm{~nm}$ and a height of $330 \mathrm{~nm}$. The crystal size is thus comparable to the actual beam size and the measurements are highly sensitive to any misalignments, e.g. with respect to the centre of rotation. The retrieved phase reveals a radial gradient of about 2 radians from the particle centre towards the crystal surface which is apparent throughout the whole thickness of the $\mathrm{Au}$ crystal, corresponding to a displacement gradient of $-0.75 \AA$.

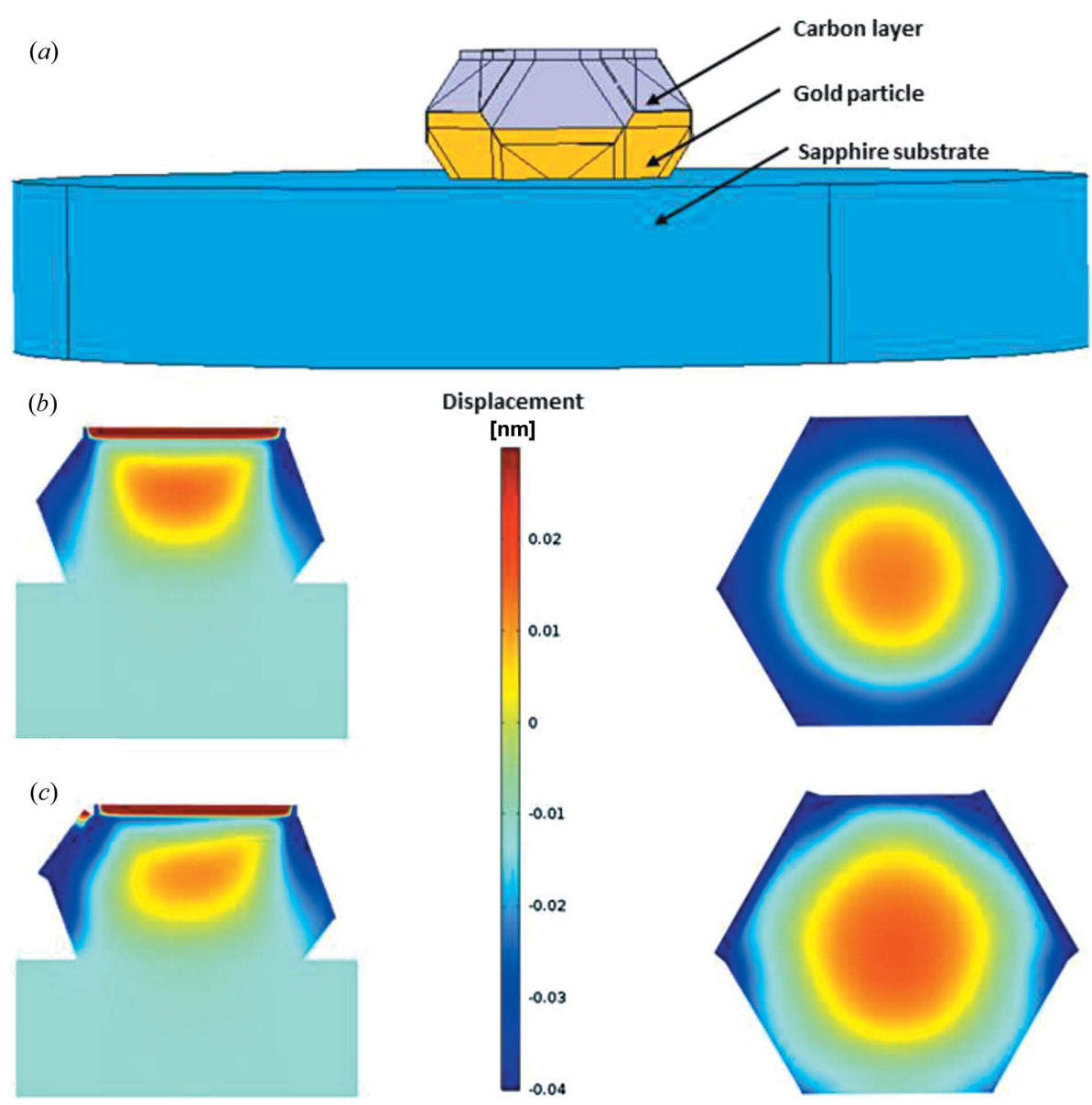

Figure 6

(a) FEM simulations of the displacement field in an $\mathrm{Au}$ crystal covered by an inhomogeneous strained carbon layer deformed by $-0.5 \%$. (b) Vertical and horizontal cuts through the 3D $z$-displacement field considering a strained carbon layer with a thickness of $30 \mathrm{~nm}$ on the crystal top facet and a thickness of $5 \mathrm{~nm}$ on the side facets. (c) Vertical and horizontal cuts through the 3D $z$-displacement field considering a strained carbon layer with a thickness of $30 \mathrm{~nm}$ on the crystal top facet, a thickness of $5 \mathrm{~nm}$ on one half of the side facets and an increased thickness of $30 \mathrm{~nm}$ on the other half of the side facets.
A similar phase gradient was observed for highly inhomogeneously strained $\mathrm{Si}$ lines on $\mathrm{SiO}_{2} / \mathrm{Si}$ substrates (Minkevich et al., 2007). It shows the presence of a strained layer on top of the Au island. Such a layer could be carbon (as illustrated by Fig. S2) that is deposited on top of the Au particle by the highly focused and intense X-ray beam during the measurement. This carbon deposition may originate either from the cracking of hydrocarbons by photoelectrons emitted from the nano-object, which was also reported for Au nanowires (Shin et al., 2018) and for Ag/Au core-shell nanowires (Haag et al., 2013), or from residuals of the photoresist used for the lithographic patterning, which is carbonized by the X-ray beam.

The phase field and thus the displacement field vary from the BCDI to the mw-BCDI, which may be caused by the inhomogeneity of the deposited carbon. In fact, the crystal illumination by the X-ray beam while scanning is different for the two techniques. The goniometer wobble and slight offset of the sample with respect to the centre of rotation induces a 'sweeping' effect which leads to a rather homogeneous illumination of the crystal when rocking the sample. Conversely, in the case of mw-BCDI the incident beam always illuminates the same sample area. When the size of the incident beam is roughly the same as the particle size, minutely off-centre positioning of the sample with respect to the beam focus leads to more photons and thus more carbon deposition on one part of the crystal than on others.

The displacement field in an $\mathrm{Au}$ crystal covered by an inhomogeneous strained carbon layer was calculated by FEM simulations. The computed sample is illustrated in Fig. 6(a). Considering a strained carbon layer deformed by $-0.5 \%$ with a thickness of $5 \mathrm{~nm}$ on the side facets and a thicker layer of $30 \mathrm{~nm}$ on the top facet of the $\mathrm{Au}$ crystal results in a cylindrical displacement field of up to $0.02 \mathrm{~nm}$ in the crystal centre, which decreases to $-0.04 \mathrm{~nm}$ towards the crystal side facets [Fig. 6(b)]. This displacement field gradient of $-0.65 \AA$ over the complete crystal is in qualitative agreement with the BCDI findings showing a gradient of $-0.75 \AA$ [Figs. 5(a) and 5(c)]. The FEM simulations also show an elevated displacement field at the edges and close to the side facets of the Au crystal, which was not detected experimentally, probably because of the voxel size of about $6^{3} \mathrm{~nm}^{3}$ and because the Au crystal has rather rounded edges instead of atomically sharp edges. When increasing the carbon layer thickness on the facets of one side of the crystal 
(which represents the direction of the incident X-ray beam and thus a preferential carbon deposition) the displacement field in the Au crystal varies [Fig. 6(c)] similarly to that found for mw-BCDI [Figs. 5(b) and 5(d)]. These FEM results are in qualitative agreement with the experimental findings, demonstrating that a thin strained carbon layer may affect the displacement field in a nanostructure and that additional carbon deposition over the course of an experiment may modify the measured phase field.

The differences in the phase fields reconstructed from the interpolated data for both scanning methods were quantified on a pixel-by-pixel basis. Considering the evolution of the sample under the X-ray beam, this comparison gives a lower limit of the actual resolution. Firstly, a phase shift has been applied on the 3D reconstructed phase values obtained from the mw-BCDI so that the central pixel has the same value as the one inferred by classic BCDI. Then, both matrices were subtracted from each other (BCDI minus mw-BCDI). The dispersion of the differences in the reconstructed phase values, illustrated by the histogram shown in Fig. 7, follows a Gaussian. The standard deviation is $0.41 \mathrm{rad}$, which corresponds to a displacement of $14 \mathrm{pm}$, indicating that both reconstructions are in rather good agreement considering the evolution of the sample during the measurements by carbon deposition. This value seems to be large compared with the accuracy of $1 \mathrm{pm}$ given by Labat et al. (2015). However, while the latter is an averaged phase shift between two extended domains, the results presented in this work correspond to the real phase accuracy within a voxel of $6^{3} \mathrm{~nm}^{3}$.

To the best of our knowledge, so far only Cha et al. (2016) have reported the reconstruction of both the Bragg electron density and the phase from coherent X-ray diffraction data recorded by scanning the photon energy of the incident coherent X-ray beam. While the authors applied a slice-byslice method, which is rather time consuming and computationally intensive, we demonstrated in the present work the

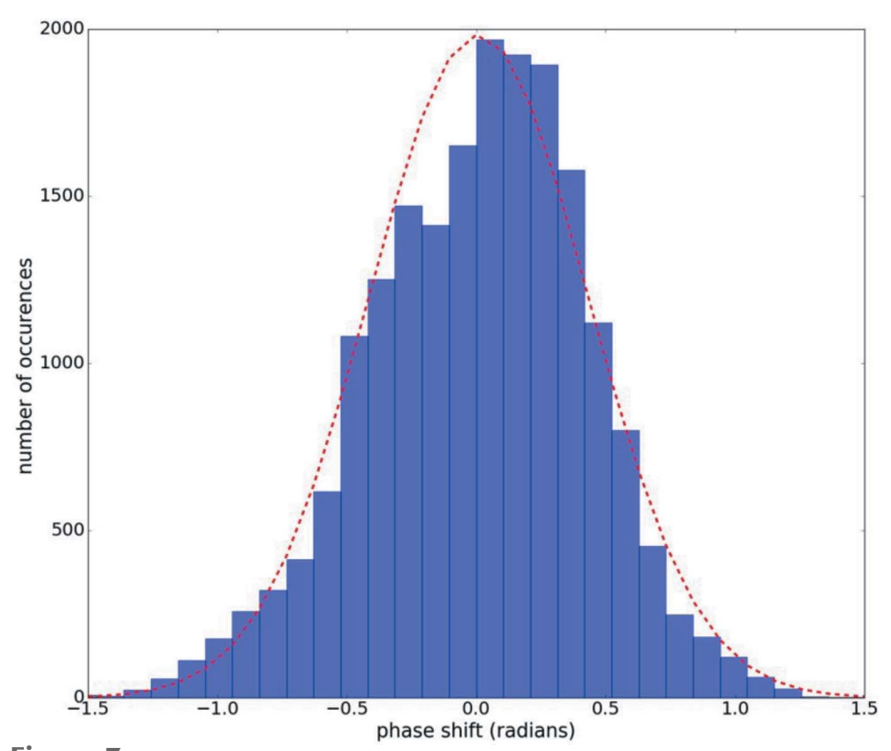

Figure 7

Histogram of the shift in phase values pixel-by-pixel between the two scanning methods. phase retrieval on interpolated data as well as on raw data using well established phase-retrieval algorithms. The two approaches used in the present work are very robust and give essentially the same result. However, it should be noted that post-interpolation leads to an increased voxel size as well as to artefacts in the retrieved phase. Pre-interpolation should thus be favoured, mitigating the variation of the effective pixel size in mw-BCDI. Minor variations of the phase field observed in mw-BCDI compared with rocking scan BCDI are probably caused by the X-ray beam induced deposition (XBID) of a carbon layer on the Au particle. This hypothesis is supported by FEM simulations, which show similar displacement fields to the experiment when considering an inhomogeneous strained carbon layer covering the Au crystal. These results underline the high sensitivity of the technique and the necessity to work under as clean conditions as possible to avoid any XBID contamination of the sample under investigation. They further illustrate that a variation of the photon energy of $\pm 2.5 \%$, which induces a dilation of the effective pixel size of the same order, does not have a major impact on the phase-retrieval process using well established algorithms. They thus represent a major step forward to actual in situ BCDI studies where the sample environment or other experimental constraints do not allow for rotating the sample stage, making mw-BCDI the only available option. This approach also offers stable illumination conditions, thus excluding any possible artefacts originating from the eccentricity of diffractometer goniometers and making additional alignments during rocking scans dispensable. As illustrated by Fig. S3, standard rocking scans induce displacements of the Au crystal of $0.83 \mu \mathrm{m}$ per degree, thus moving it out of the X-ray beam focal spot without any additional alignments.

\section{Conclusions}

Multi-wavelength Bragg coherent X-ray diffraction imaging was demonstrated on a selected Au particle, giving essentially the same result as for rocking scans. Conventional phaseretrieval algorithms combined with pre-interpolation that mitigates the dilation of the effective pixel size allow for reconstruction of the Bragg electron density and the phase field. Voxel sizes of about $6^{3} \mathrm{~nm}^{3}$ and shifts in the phase field of 0.41 radians that translate to a strain resolution of better than $10^{-4}$ were obtained for both classic and mw-BCDI. The reconstructed phase field revealed a radial gradient which extends through the whole thickness of the $300 \mathrm{~nm}$-thick crystal. This phase gradient probably originates from a strained carbon layer which was unintentionally deposited on top of the Au crystal. Differences observed in the reconstructed phase field for BCDI and mw-BCDI are attributed to slight changes in the thickness of the carbon layer induced by the X-ray beam over the course of the experiment.

The fact that mw-BCDI allows us to study immobile nanostructures avoids any sources of vibration that may be detrimental to sample environments and circumvents any risk of the nanostructure moving out of the X-ray beam focus during sample rotation owing to the inevitable wobble of a 
diffractometer. This technique thus seems pre-destined for in situ and operando studies of functional materials. The combination of conventional phase-retrieval algorithms with pre-interpolation simplifies the phase-retrieval process compared with more computationally intensive approaches and thus opens this technique to a broader community.

\section{Acknowledgements}

The authors gratefully acknowledge both the European Synchrotron Radiation Facility for beamtime allocation at the ID01 beamline and the beamline staff for excellent support during the measurement campaign. The authors further acknowledge J. Carnis from IM2NP in Marseille for fruitful discussions. The thin-film processing was performed at the Micro-Nano Fabrication and Printing Unit (MNF\&PU), Technion.

\section{References}

Biermanns, A., Davydok, A., Paetzelt, H., Diaz, A., Gottschalch, V., Metzger, T. H. \& Pietsch, U. (2009). J. Synchrotron Rad. 16, 796802.

Cha, W., Ulvestad, A., Allain, M., Chamard, V., Harder, R., Leake, S. J., Maser, J., Fuoss, P. H. \& Hruszkewycz, S. O. (2016). Phys. Rev. Lett. 117, 225501.

Chahine, G. A., Richard, M.-I., Homs-Regojo, R. A., Tran-Caliste, T. N., Carbone, D., Jacques, V. L. R., Grifone, R., Boesecke, P., Katzer, J., Costina, I., Djazouli, H., Schroeder, T. \& Schülli, T. U. (2014). J. Appl. Cryst. 47, 762-769.

Chahine, G. A., Zoellner, M. H., Richard, M.-I., Guha, S., Reich, C., Zaumseil, P., Capellini, G., Schroeder, T. \& Schülli, T. U. (2015). Appl. Phys. Lett. 106, 071902.

Clark, J. N., Ihli, J., Schenk, A. S., Kim, Y.-Y., Kulak, A. N., Campbell, J. M., Nisbet, G., Meldrum, F. C. \& Robinson, I. K. (2015). Nat. Mater. 14, 780-784.

Cornelius, T. W., Davydok, A., Jacques, V. L. R., Grifone, R., Schülli, T., Richard, M.-I., Beutier, G., Verdier, M., Metzger, T. H., Pietsch, U. \& Thomas, O. (2012). J. Synchrotron Rad. 19, 688-694.

Davydok, A., Cornelius, T. W., Ren, Z., Leclere, C., Chahine, G., Schülli, T., Lauraux, F., Richter, G. \& Thomas, O. (2018). QuBS, 2 , 24.

Diaz, A., Mocuta, C., Stangl, J., Mandl, B., David, C., Vila-Comamala, J., Chamard, V., Metzger, T. H. \& Bauer, G. (2009). Phys. Rev. B, 79, 125324.

Dupraz, M., Beutier, G., Cornelius, T. W., Parry, G., Ren, Z., Labat, S., Richard, M.-I., Chahine, G. A., Kovalenko, O., De Boissieu, M., Rabkin, E., Verdier, M. \& Thomas, O. (2017). Nano Lett. 17, 66966701.

Favre-Nicolin, V., Eymery, J., Koester, R. \& Gentile, P. (2009). Phys. Rev. B, 79, 795401.

Favre-Nicolin, V., Mastropietro, F., Eymery, J., Camacho, D., Niquet, Y. M., Borg, B. M., Messing, M. E., Wernersson, L.-E., Algra, R. E., Bakkers, E. P. A. M., Metzger, T. H., Harder, R. \& Robinson, I. K. (2010). New J. Phys. 12, 035013.

Fienup, J. R. (1978). Opt. Lett. 3, 27-29.
Fienup, J. R. (1982). Appl. Opt. 21, 2758-2769.

Gerchberg, R. W. \& Saxton, W. O. (1972). Optik, 35, 237.

Haag, S. T., Richard, M. I., Welzel, U., Favre-Nicolin, V., Balmes, O., Richter, G., Mittemeijer, E. J. \& Thomas, O. (2013). Nano Lett. 13, 1883-1889.

Jacques, V. L. R., Ravy, S., Le Bolloc'h, D., Pinsolle, E., SauvageSimkin, M. \& Livet, F. (2011). Phys. Rev. Lett. 106, 065502.

Kovalenko, O., Greer, J. R. \& Rabkin, E. (2013). Acta Mater. 61, 3148-3156.

Kovalenko, O. \& Rabkin, E. (2015). Scr. Mater. 107, 149-152.

Labat, S., Richard, M.-I., Dupraz, M., Gailhanou, M., Beutier, G., Verdier, M., Mastropietro, F., Cornelius, T. W., Schülli, T. U., Eymery, J. \& Thomas, O. (2015). ACS Nano, 9, 9210-9216.

Leake, S. J., Chahine, G. A., Djazouli, H., Zhou, T., Richter, C., Hilhorst, J., Petit, L., Richard, M.-I., Morawe, C., Barrett, R., Zhang, L., Homs-Regojo, R. A., Favre-Nicolin, V., Boesecke, P. \& Schülli, T. U. (2019). J. Synchrotron Rad. 26, 571-584.

Leake, S. J., Favre-Nicolin, V., Zatterin, E., Richard, M.-I., Fernandez, S., Chahine, G., Zhou, T., Boesecke, P., Djazouli, H. \& Schülli, T. U. (2017). Mater. Des. 119, 470-471.

Luke, D. R. (2005). Inverse Probl. 21, 37-50.

Mandula, O., Elzo Aizarna, M., Eymery, J., Burghammer, M. \& FavreNicolin, V. (2016). J. Appl. Cryst. 49, 1842-1848.

Marchesini, S., He, H., Chapman, H. N., Hau-Riege, S. P., Noy, A., Howells, M. R., Weierstall, U. \& Spence, J. C. H. (2003). Phys. Rev. $B, \mathbf{6 8}, 140101$.

Miao, J. W., Charalambous, P., Kirz, J. \& Sayre, D. (1999). Nature, 400, 342-344.

Minkevich, A. A., Gailhanou, M., Micha, J.-S., Charlet, B., Chamard, V. \& Thomas, O. (2007). Phys. Rev. B, 76, 104106.

Neighbours, J. R. \& Alers, G. A. (1958). Phys. Rev. 111, 707-712.

Newton, M. C., Leake, S. J., Harder, R. \& Robinson, I. K. (2010). Nat. Mater. 9, 120-124.

Nishino, Y., Takahashi, Y., Imamoto, N., Ishikawa, T. \& Maeshima, K. (2009). Phys. Rev. Lett. 102, 018101.

Pfeifer, M. A., Williams, G. J., Vartanyants, I. A., Harder, R. \& Robinson, I. K. (2006). Nature, 442, 63-66.

Pfeiffer, F. (2018). Nat. Photon. 12, 9-17.

Ponchut, C., Rigal, J. M., Clément, J., Papillon, E., Homs, A. \& Petitdemange, S. (2011). J. Instrum. 6, C01069.

Ren, Z., Mastropietro, F., Davydok, A., Langlais, S., Richard, M.-I., Furter, J.-J., Thomas, O., Dupraz, M., Verdier, M., Beutier, G., Boesecke, P. \& Cornelius, T. W. (2014). J. Synchrotron Rad. 21, 1128-1133.

Richard, M.-I., Fernandez, S., Eymery, J., Hofmann, J. P., Gao, L., Carnis, J., Labat, S., Favre-Nicolin, V., Hensen, E. J. M., Thomas, O., Schülli, T. \& Leake, S. J. (2018). Nanoscale, 10, 4833-4840.

Robinson, I. K., Vartanyants, I. A., Williams, G. J., Pfeifer, M. A. \& Pitney, J. A. (2001). Phys. Rev. Lett. 87, 195505.

Shin, J., Cornelius, T. W., Labat, S., Lauraux, F., Richard, M.-I., Richter, G., Blanchard, N. P., Gianola, D. S. \& Thomas, O. (2018). J. Appl. Cryst. 51, 781-788.

Thibault, P., Elser, V., Jacobsen, C., Shapiro, D. \& Sayre, D. (2006). Acta Cryst. A62, 248-261.

Ulvestad, A., Clark, J. N., Harder, R., Robinson, I. K. \& Shpyrko, O. G. (2015a). Nano Lett. 15, 4066-4070.

Ulvestad, A., Clark, J. N., Singer, A., Vine, D., Cho, H. M., Harder, R., Meng, Y. S. \& Shpyrko, O. G. (2015b). Phys. Chem. Chem. Phys. 17, 10551-10555.

Williams, G. J., Pfeifer, M. A., Vartanyants, I. A. \& Robinson, I. K. (2003). Phys. Rev. Lett. 90, 175501. 